\title{
DEFINING HOSPITAL CLUSTERS AND ASSOCIATED SERVICE COMMUNITIES IN METROPOLITAN AREAS $\dagger$
}

\author{
J. WILLIAM THOMAS \\ Department of Medical Care Organization, School of Public Health, University of Michigan, Ann Arbor, MI 48104, \\ U.S.A. \\ and \\ JOHN R. GRIFFITH and PAUL DURANCE \\ Program and Bureau of Hospital Administration, School of Public Heaith, University of Michigan, Ann Arbor, \\ MI 48104, U.S.A.
}

\author{
(Received 26 September 1980)
}

\begin{abstract}
According to traditional concepts of hospital governance, each institution is considered responsible for the care of a defined community. Evaluation of hospital performance and effective service planning both require that hospitals' service communities be identified. However, in metropolitan regions it is difficult to associate a geographic population with any one hospital because of the wide choice of facilities available to area residents. The service community concept becomes more meaningful in these regions if several hospitals with overlapping geographic communities are defined as a cluster.

This paper describes a two-step method for identifying hospital clusters and their associated service communities. The first step involves analysis of patient origin data to identify logical clusters of hospitals. Three algorithms for performing this analysis are presented. In the second step, analytical findings are reviewed by a panel of area planners and hospital experts who, considering additional qualitative factors, determine how the hospitals may be most appropriately grouped. Experience in using this method for hospitals in the seven-county region around Detroit, Michigan suggests that reasonable clusters can be identified, although clusters of central city hospitals are less well-defincd than those in the suburban areas.
\end{abstract}

The central purpose of this country's nearly $6000 \mathrm{com}$ munity hospitals traditionally has been to provide care to residents of the hospitals' home communities. Although regional coordination of decisions concerning hospital size, location and services has been stressed since the late 1960 s and emphasized particularly since the passage of PL 93-641 in 1974, governance of community hospitals remains a local, rather than regional, responsibility.

At the local level, effective decision-making by a hospital's governing board depends to a great degree upon the board's knowledge of the needs of the hospital's service community and this, in turn, requires that the service community be clearly identified and measured. As stated by the American Hospital Association, "careful appraisal by each institution of the area and people it plans to serve is essential to effective personal health services planning" [1].

In regions which contain few, geographically dispersed hospitals, identification of the population served by each hospital is reasonably straightforward. $\ddagger$ However, in metropolitan regions patients typically have numerous hospitals available within reasonable travel distance. When selecting hospitals for care, patients in these regions are often influenced by ethnic and religious factors, medical staff affiliations of their physicians, physician referral patterns, availability of special services at particular hospitals and other non-spatial considerations [3,5-8]. Thus the concept of each in-

tThe research described in this paper was supported through a grant from the W. K. Kellogg Foundation. Additional details on the research, including algorithms used, are available in the Hospital Performance Measures Project, University of Michigan Program and Burcau of Hospital Administration, Technical Paper Number 5.

$\ddagger$ See, e.g. $[2,4]$. stitution possessing its own distinct service area is rarely meaningful for metropolitan hospitals.

Nevertheless, evidence suggests that geography remains an important concern in these patient decisions [9]. Residents of a suburban community are more likely to utilize hospitals located in or near that suburb than to travel to central-city hospitals. In few cases do patients leave a relatively self-sufficient group of hospitals in one suburban area to seek hospital care in another suburb. And city residents tend to use city hospitals, with some preference given to hospitals located in the general vicinity of the patients' residences.

Where several adjacent neighborhoods, small suburbs or other areas located within an urban region are served by a common set of hospitals, the set of hospitals can be defined as a cluster, with the combined areas constituting the cluster's service community. While this cluster concept does not provide for identification of populations uniquely associated with individual hospitals, it does allow meaningful service communities to be defined for groups of hospitals and it can demonstrate the necessity for coordinated organization and delivery of services by neighboring institutions.

The purpose of this paper is to present an approach for defining hospital clusters and their service communities. Methods to test the existance of hospital groups serving overlapping geographic communities (clustering) will be reviewed. Using data from a statewide patient origin study, clustering methods are applied to hospitals in the metropolitan area around Detroit, Michigan. This region contains 82 hospitals and is composed of seven counties with populations ranging from 82,000 to 2.48 million. Identification of hospital clusters and service communities was the first step in a multi-year project to evaluate the impact of presenting annual evaluative data on hospital performance to areawide planners, hospital 
administrators and hospital trustees. With cluster service communities defined, measures can be obtained which relate performance to size and other characteristics of the communities being served. $\dagger$

\section{CLUSTER DEFINITION METHODS}

A cluster will contain one or more hospitals which (together) account for most or all of the hospital care provided to residents of an identifiable community. For a well-defined cluster, it can be shown based on objective analysis of patient origin data that patients from the geographic community surrounding the group of hospitals are likely to use one or more of those facilities for care. Additionally, the grouping should be considered generally consistent with known qualitative factors such as: formal and informal cooperative efforts among hospitals, inter-hospital competition, general community perceptions of hospital service areas and other commercial and political commonalities.

Consistent with above characteristics, a two-step approach to the specification of clusters was formulated. The first step of the clustering process requires analysis of patient origin data to identify groupings of area hospitals that are most appropriate in terms of actual patterns of patient use. Three such techniques-the greedy algorithm, the max-flow/min-cut algorithm and the maxrelevance algorithm-are discussed below.

The second step in the process is to submit the analytical results to a cluster review committee, a group of experts familiar with the local area. Based on members' knowledge of hospital relationships and other factors influencing the reasonableness of proposed groupings, the committee is asked to decide whether the objectively determined clusters are in fact appropriate. Where several alternative groupings are equally plausible in terms of the objective criterion, committee members are asked to select the one that in their opinion is the most reasonable. Other adjustments, such as transferring a hospital from one cluster to another, may also be made by the committee. Thus the committee makes the final determination, using the patient origin data analysis as one important source of information.

\section{Analytic methods}

A number of methods described in the literature have been used for partitioning large regions, e.g. states or sub-state regions, into smaller non-overlapping areas such as hospital districts, health planning areas or medical service areas[11-25]. Generally, regions to be partitioned are composed of numerous small areal units such as census tracts, and each district of a region can be defined as a contigous subset of the region's areal units. Several of the methods for defining districts may be applied to the problem of identifying hospital clusters, since hospitals located within a district could constitute a cluster and residents of the district then would make up that cluster's service community.

Techniques described by Poland and Lembke[16], Ciocco and Altman[12], Taliaferro and Remmers [24], Thomas [20] and Transaction Systems, Inc.[19], utilize conceptually similar approaches for defining district boundaries. With each of these methods, several areal

tFor a discussion of performance measures and an overview of the project, see Griffith[10]. In addition to the Southeastern Michigan Region, the project also focuses on Michigan's six other Health Services Areas. The procedures described in this paper for clustering Southeastern Michigan hospitals were also employed in these other areas. units in the region (those containing hospitals and/or having a net inflow of patients above a specified level) are selected to be district centers. The remaining areal units are then assigned, one by one, to districts. While these techniques differ in such respects as the types of patient-use data considered, the types of areal units employed and whether or not individual areal units can be split or reassigned, all utilize basically the same logic for assigning areal units to districts. The rule for selecting the next areal unit for assignment may be termed the "greedy" heuristic, since it selects the unit whose assignment appears, at that point, to offer the greatest reduction in cross-district-boundary patient traffic; that is, the areal unit and developing district that share the greatest amount of patient traffic are identified, and the areal unit is assigned to that district. Taliaferro and Remmers, Thomas and Transaction Systems, Inc. utilize computer-based algorithms for data analysis and district definition, while Ciocco and Altman's and Poland and Lembke's procedures were performed manually. DeVise, in defining hospital planning districts for Chicago, employed an approach similar to that of Ciocco and Altman[15]. Related procedures were also utilized by Gittelsohn and Wennberg[4] in Vermont and the Citizens' Hospital Study Committee [18] in Northeastern Ohio.

Instead of building up districts through sequential assignment of areal units, the max-flow/min-cut procedure proposed by Thomas [20] divides a region into ever-smaller pieces. As a first step, the region (consisting, say, of $N$ zip codes) is divided along zip code boundaries into two districts. Another cut is then made to yield three districts; the next cut yields four districts, etc. This process continues until a user-specified number of cuts are completed. With this algorithm, each areal unit in the region is considered to represent one node of a network and the capacity of the arc connecting areal units $i$ and $j$ in the network is defined to be the patient traffic, or flow, between $i$ and $j$. Ford and Fulkerson's [26] max-flow/min-cut theorem then provides a basis for locating optimal cuts.

Griffith's [2] relevance index method, unlike the techniques discussed above, is used for defining service populations of individual hospitals rather than dividing regions into districts. With this method, the size of a hospital's service population is determined by (a) multiplying each areal unit's total population times the percentage of patients from the areal unit who utilize that hospital and the (b) summing these figures over all areal units in the area. (Bailey [27] in 1952 labelled this the hospital's "total effective population.") The key measure in these calculations, the percentage of patients in an areal unit who use the hospital, is called "relevance index". Although the relevance index method was intended only for calculating service communities of individual hospitals, a straightforward extention of the procedure may be used for defining hospital clusters. In areas containing several hospitals, relevance index values can be improved by selectively grouping hospitals into clusters, and the degree of improvement achieved can serve as a guide when determining which hospitals to cluster together.

The first step of this modified procedure, which shall be termed the max-relevance algorithm, is to calculate a population-weighted average relevance index $\bar{R}_{j}$ for each hospital. Letting: $P_{i}=$ population of areal unit $i ; d_{i j}=$ number of patients from areal unit $i$ treated at hospital $j$; $D_{i}={ }_{j}^{5} d_{i j}=$ total patients from areal unit $i ; I_{i}=$ $\left\{i \mid\left(d_{i j} j D_{i}\right) \geq \alpha\right\}$, set of areal units for which individual relevance values $\left(d_{i j} / D_{i}\right)$ of hospital $j$ exceeds or equals $\alpha$, where $\alpha$ is specified $0 \leq \alpha \geqslant 1$; Then $\bar{R}_{j}=$ 
$\sum_{i \in I_{j}} P_{i}\left(d_{i j} / D_{i}\right) / \sum_{i \in I_{j}} P_{i}$. After $\bar{R}_{i}$ is calculated for each individual hospital, the hospital with the smallest $\bar{R}_{\mathrm{j}}$ is identified and grouped to form a cluster with the hospital having the greatest individual relevance in hospital $j$ 's home areal unit. A new value of $\bar{R}_{j}^{* *}$ is determined as above, where $j^{*}$ refers to the two-hospital cluster. Values of $\vec{R}_{i}$ (for non-cluster hospitals) and $\bar{R}_{*^{*}}$ (for clusters) are scanned to locate the minimum again. As before, the hospital (or cluster) with the smallest average relevance index is identified for clustering and is grouped with the hospital (or cluster) having the greatest individual relevance in the identified hospital's home areal unit. (When a previously formed cluster $j^{*}$ is identified for further clustering, its home areal unit is assumed to be the home areal unit of the hospital (member of $j^{*}$ ) having the highest $R_{i j}$ among the cluster hospitals' home areas.) At each iteraction, a new hospital or cluster is selected and grouped with another hospital or cluster. The procedure terminates when one of three conditions occurs: (1) all hospitals have been aggregated into a single large cluster; (2) a user-specified number of iterations has been completed; or (3) all identified clusters are stable, i.e. no cluster serves more than $\alpha$ of the patients in the home areal unit of any other cluster.

\section{ANALYSIS AND RESULTS}

A greedy algorithm and the max-flow/min-cut and maxrelevance algorithms were each programmed in $\mathrm{PL} / 1$ for analysis of Southeastern Michigan patient origin data. Analyses were run on the University of Michigan's Amdahl 470/V6 computer. The following sections describe the data hase used for the analyses, results of the analyses including difficulties encountered in clustering central city Detroit hospitals, and use of the analytical results by a panel of local hospital experts.

\section{Data}

Beginning in 1975, various planning agencies and provider associations with interests in hospital care in Michigan began collecting standarized patient discharge abstract data for Michigan hospitals. These data describe, for each Michigan hospital, the number of obstetrical, pediatric, surgical and non-surgical discharges during the year. Discharge data are further broken down by patient residence zip code for patients residing in Michigan and by state for residents of states adjoining Michigan. Although discharges are classified in the data as to hospital service (obstetrical, pediatric, etc.), only discharge totals, broken down by patient residence zip code, were used in the study. The final data base for the study included 600,000 discharges from hospitals located in the Southeastern Michigan area.

\section{Analysis}

Initial attempts to define hospital clusters throughout the 174 zip code region were unsuccessful. After five minutes of cpu time, the max-flow/min-cut algorithm had managed to complete only three cuts. While the greedy and maxrelevance algorithms were able to yield solutions in less than $22 \mathrm{sec}$. of cpu time, the compactness and relative size of clusters defined for Detroit and adjacent suburbs were considered unsatisfactory.

Problem size for the max-flow/min-cut algorithm is determined primarily by the number of node-to-node (residence zip code to hospital zip code) connections defined in the patient origin data. In a densely populated area like Detroit, residents of a neighborhood are likely to utilize a number of different hospitals and thus the number of patient flow connections among city zip codes is quite large. Problems encountered by the greedy and maxrelevance algorithms were traceable to the same phenomenon. A few major hospitals located in close proximity to each other attract large numbers of patients from diverse parts of the city, and as a consequence the algorithms tended to form very large clusters in the lowntown area.

'To make the analysis by the max-flow/min-cut and greedy algorithms more tractable, each algorithm was applied independently to the 84 zip codes comprising Detroit and its close suburbs and to the 90 zip codes making up the outlying region. Both algorithms identified five strong clusters of hospitals in the outlying area, with max-flow/min-cut using $7.7 \mathrm{cpu}$ seconds and the greedy only 3.7 seconds. For service areas defined, $94 \%$ of patients utilized associated cluster hospitals. However, neither algorithm was able to locate an acceptable solution for the Detroit hospitals. Solutions for Detroit contained a number of single hospital clusters and one excessively large cluster containing approximately 30 hospitals and 60 zip code areas. Other computer runs were made, some with certain of the larger hosptials removed from the data, but problems with enclaves (single hospital areas embedded in larger cluster areas) and unacceptably large cluster areas in the central city continued to occur.

Max-relevance algorithm results were observed during initial runs to be extremely sensitive to the value of the parameter $\alpha$. Runs with low $\alpha$ values $(0.01$ and smaller) yielded several large clusters. High values of $\alpha$ produced many small clusters; several clusters in the outlying region differed from the min-cut and greedy algorithms' solutions and those in the suburban and inner Detroit areas appeared unreasonable in light of known patient use patterns. At each step, the max-relevance algorithm selects the hospital (or cluster) having the lowest $\bar{R}_{j}$ (or $\bar{R}_{j^{*}}$ ) and adds it to another hospital or cluster. If hospitals $j$ and $k$ each have large numbers of low relevance zip codes included in the sets $I_{j}$ and $I_{k}$ (and therefore have low values of $\bar{R}_{j}$ and $\bar{R}_{k}$ ), the cluster formed by grouping these hospitals may have a relevance index value lower than both $\bar{R}_{j}$ and $\vec{R}_{k}$. As illustrated in Fig. 1, with $\alpha$ set at $0.02, I_{j}$ includes zip codes for which hospital $k$ has zero (or less than $2 \%$ ) relevance. Similarly, $I_{k}$ includes zip codes for which hospital $j$ has zero relevance. If the set $I_{j^{*}}$ for the cluster of $j$ and $k$ is defined as the union of $I_{i}$ and $I_{k}, \bar{R}_{j^{*}}$ will necessarily be lower than either $\bar{R}_{j^{*}}$ or $\bar{R}_{k}$, Thus the cluster of $j$ and $k$ would be selected for additional clustering at the next iteration and $\bar{R}_{j^{*}}$ could be reduced further. This expanded cluster would in turn be selected at the next iteration, etc. By modifying the algorithm to allow two $\alpha$ levels, $\alpha=0.125$ for clusters of two or more hospitals and $\alpha=0.02$ for single hospitals, this problem was substantially reduced. After the modification, the max-relevance algorithm was able to

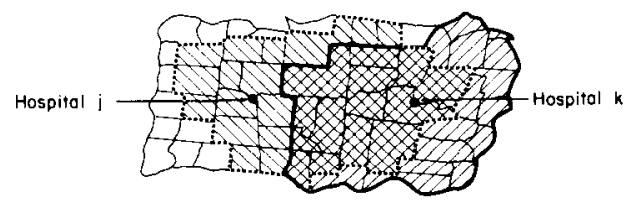

$$
\begin{aligned}
& \text { ZIP Code areas in } I_{i} \text { only } \\
& \text { ZIP code areas in } I_{k} \text { only } \\
& \text { ZIP Code areas in both } I_{j} \text { and } I_{k}
\end{aligned}
$$

Fig. 1. Illustration of overlapping of hospital service areas. 
define nineteen clusters for the seven county region. Each of the defined clusters in the far suburbs and outlying region included many zip code areas with more than $50 \%$ relevance to cluster hospitals. As shown in Fig. 2 , clusters were similar to those defined by the greedy and min-cut algorithms. (Note that the greedy and mincut solutions include fixed service area boundaries while the max-relevance solution does not.)

Unlike the other algorithms, the max-relevance procedure was able to locate acceptable groupings in the inner suburbs and central city area of Detroit. However, the quality of clusters (measured by average relevance index) for hospitals in and around Detroit was lower than that of the outlying clusters. In addition to the nineteen clusters identified, four alternative groupings of Detroit area hospitals were defined, each representing a slight improvement in average relevance index, but with an associated increase in cluster size. An alternative cluster specification was also identified for one of the outlying counties.

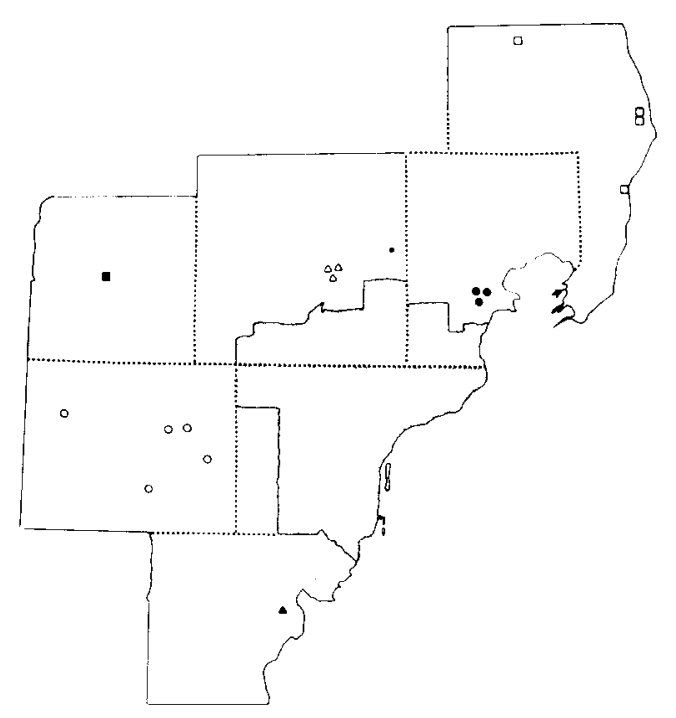

(a) Max-Relevance Algorithm Solution.

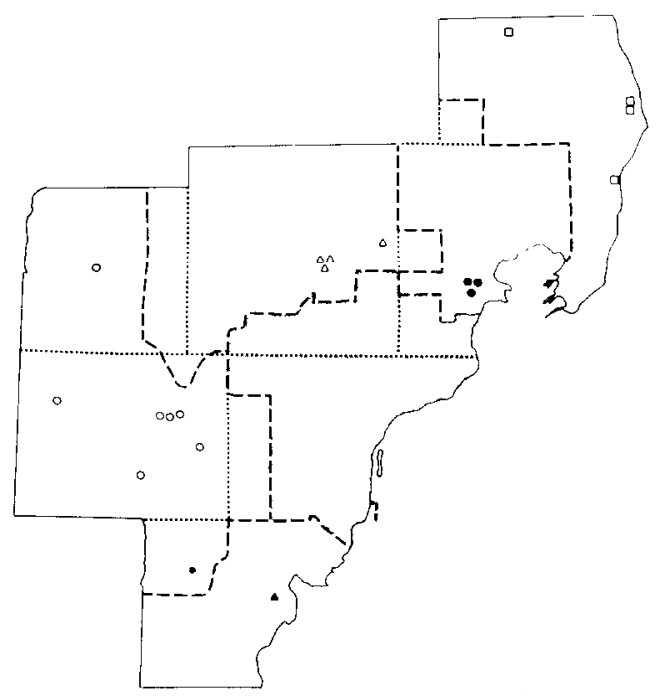

(c) Max-Flow/Min-Cut Algorithm Solution.

Fig. 2. Hospital clusters for remote suburbs in Southeastern Michigan Health Services Area.
Results of the max-relevance analysis are shown in Table 1. The primary clusters are listed, as are the five alternative groupings. Cluster service populations and average relevance index values are shown for three cases: (a) when the sets $I_{j^{*}}$ are limited to zip codes for which cluster hospitals have average relevance index values (market penetration) at or above 0.5 ; (b) when the sets $I_{j^{*}}$ are limited to zip codes for which cluster hospitals have average relevance index values at or above 0.125 ; and (c) when sets $I_{j *}$ contain all zip codes for which average relevance index values exceed zero. The last two columns show the number of zip code areas in cluster service communities $\left(I_{j^{*}}\right)$ where relevance index values equal or exceed 0.5 and 0.125 , respectively.

\section{Use of analytical results}

A cluster review panel was established jointly by the Southeast Michigan Health Systems Agency and the Greater Detroit Area Hospital Council (GDAHC) to formalize hospital groupings. Panel members, chosen

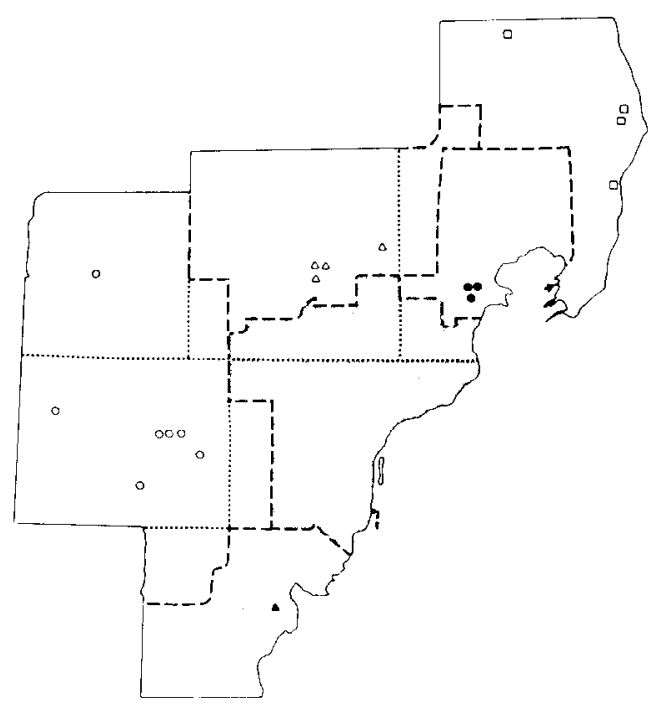

(b) Greedy Algorithm Solution.

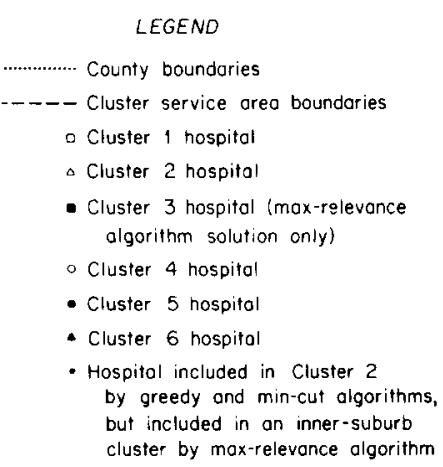




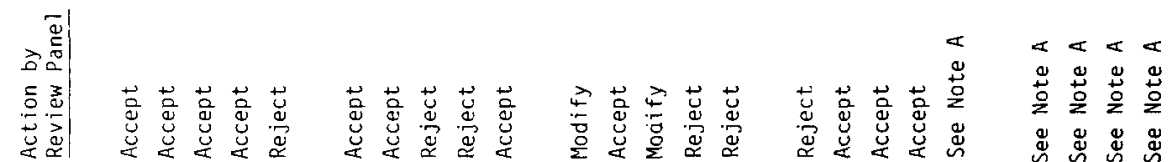

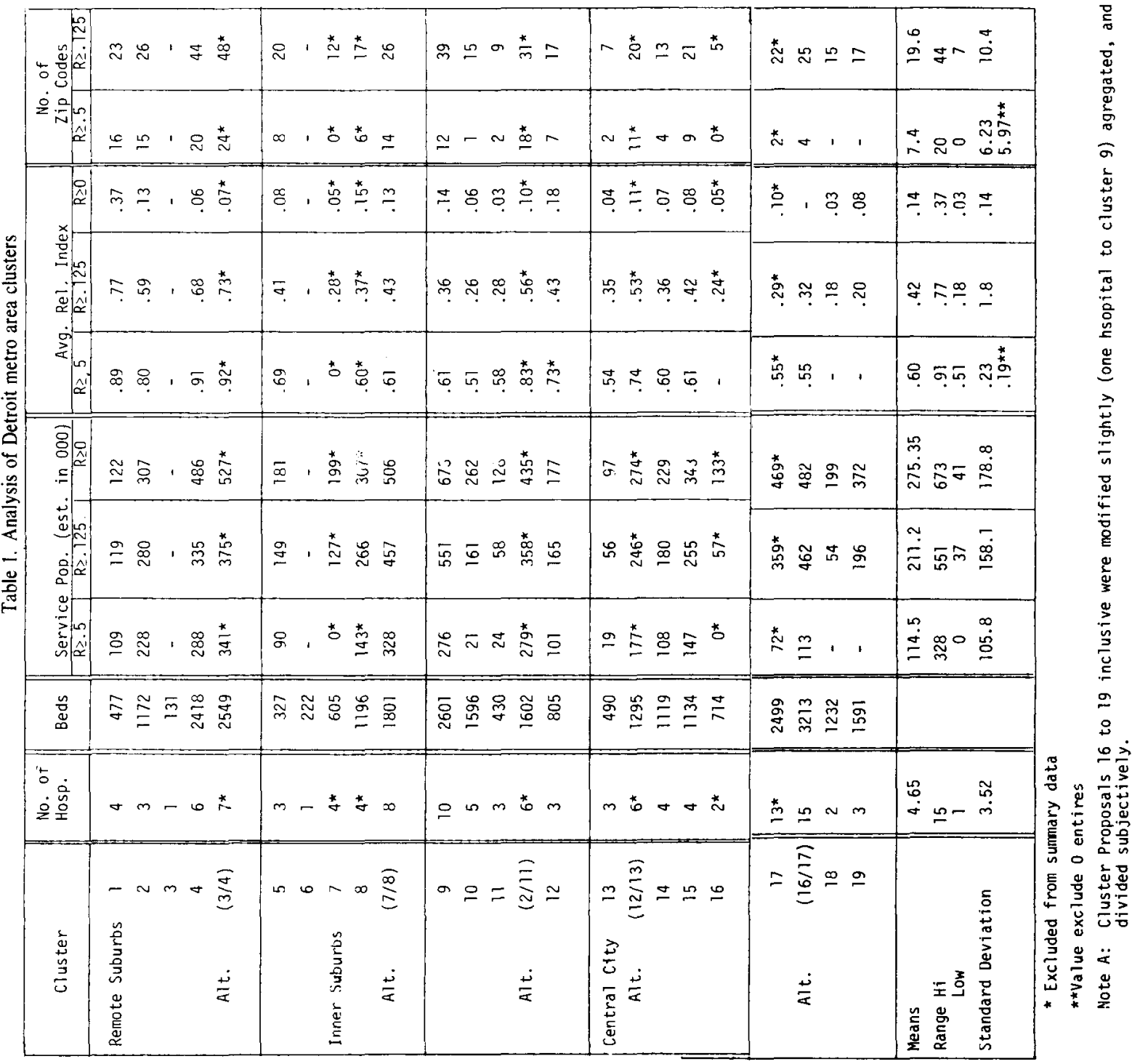


for their knowledge of hospital affairs within the region, included four representatives from area hospitals, one from the health systems agency staff, two nonprovider board members of the health systems agency and one from GDAHC. The panel's stated objective was to define hospital groupings which would facilitate coordinated and cooperative decision-making and encourage "proper" decisions from the standpoint of the region as a whole.

Prior to their meeting, the seven representatives were given the cluster recommendations shown in Table 1. (Max-relevance algorithm results were used since the greedy and min-cut algorithms did not yield acceptable clusters for the Detroit area.) At the meeting, after discussion of the analytical results the panel accepted most of the computer-generated clusters as valid. Where options were given, the panel twice selected the alternative with smaller cluster sizes, where size refers both to the number of hospitals in a cluster and to geographic distances among hospitals. Larger cluster alternatives prevailed only where the committee felt considerable subjective evidence supported them. In three cases where hospitals of multi-hospital corporations were assigned to different clusters by the max-relevance algorithm, the panel accepted the recommended clusters. In a fourth case of the same kind, the panel voted to reassign a corporate hospital to reflect a longstanding non-corporate affiliation agreement. The panel also consolidated the four central Detroit clusters (clusters 16-19, listed in Table 1) into one and defined a "sub-cluster" arrangement for this group reflecting hospital location and teaching status.

\section{LIMITATIONS OF THE ANALYSIS}

While non-quantifiable factors were considered by the review panel in its final definition of Southeastern Michigan hospital clusters, the analytical results provided the starting point for the panel's deliberations and played a major role in the decision reached. Because of the panel's reliance on the analytically defined groupings, it is important that methodological and data-related limitations of the analysis be clearly recognized.

Data age is one important concern. Although the 1975 patient origin data were the latest available, several of the 82 hospitals included in the data had closed and several new hospitals had opened. Changes in patient use patterns resulting from these systems changes had to be estimated by the review panel.

A second factor, related both to data adequacy and analytic methodology, is the HSA boundary effect. Hospitals located near the periphery of the health service area might appropriately be grouped with hospitals in an adjacent HSA. Fortunately, all boundaries of the Southeastern Michigan HSA are located in areas of low population density, and patient traffic into and out of the region is small.

The three algorithms employed for patient origin data analysis all tended to form very large clusters in the densely populated Detroit area, indicating that many hospitals there share the same (geographic) service community. While the max-relevance algorithm, after adjustment of $\alpha$, was able to suggest smaller groupings (the largest with 13 hospitals), these clusters are characterized by low relevance index values. For example, cluster 10 serves only $26 \%$ of the service community population $\left(I_{j^{*}}\right.$ defined with $\left.\alpha=0.125\right)$. Combining several of these clusters into a larger grouping of 30-40 hospitals would have improved $R_{j^{*}}$ considerably, but the resulting cluster would have been too large for meaningful description of a service community and promotion of coordination of services among hospitals. While algorithms capable of objectively considering trade-offs between high relevance index values and small cluster sizes might be developed with further research, experience now suggests that in areas such as Detroit no sets of clusters will be found that are completely satisfactory in terms of both these criteria.

\section{CONCLUSION}

Hospitals traditionally have been viewed as serving identifiable local communities. It is therefore appropriate when assessing hospital performance to evaluate how well the hospital meets the needs of its community in terms of quantity, quality and costs of services provided. However, in metropolitan areas, where a patient's choice of hospital frequently is determined by non-locational factors, rarely can meaningful service communities be defined for individual facilities. Thus, in these areas, relating individual hospital performance to community needs usually is not possible.

Analysis of patient origin data from the Detroit metropolitan area indicates that logical clusters of several neighboring hospitals can be defined such that a meaningful service community for each group of hospitals is identifiable. Further, experience in Southeastern Michigan (and in six other Michigan HSAs) suggests that hospital clusters defined through analysis of patient residence and use patterns will in most cases also be reasonable in terms of other criteria that local hospital experts might apply. While a combined service community defined for a cluster of hospitals might have less utility for service planning, hospital performance evaluation and other purposes than would discrete service communities defined for individual facilities, clustering appears to be the only realistic approach in metropolitan areas where meaningful service communities for individual hospitals generally do not exist.

In the suburbs and outlying areas of the Southeastern Michigan region, hospital clusters and their associated service communities were easily identified. The greedy and $\max$-flow/min-cut algorithms each were able to define cluster service areas for which only $6 \%$ of patients crossed area boundaries for hospital care. Similarly, in this part of the region clusters defined by the maxrelevance algorithm were strong, i.e. each cluster had a large number of associated zip code areas with high relevance to cluster hospitals. However, in the central city part of the region, the two algorithms which define explicit service areas for clusters (greedy and maxflow/min-cut) were unable to produce reasonable solutions, and clusters defined by the max-relevance algorithm were generally weak in terms of average relevance index values. Evaluations relating performance of central city hospital clusters to cluster service communities must be interpreted cautiously, given the generally weak service community definitions. Nevertheless, identification and formalization of the clusters, including those in the central city area, are expected to promote greater coordination of service planning among hospitals and increased recognition of common responsibilities for addressing the needs of their shared service populations. 


\section{REFERENCES}

1. Americal Hospital Association, Statement on Planning. AHA, Chicago (1970).

2. J. R. Griffith, Quantitative Techniques for Hospital Planning and Control. Heath, Lexington, Mass. (1972).

3. P. Zimmerman, Services areas and their needs must be reassessed. Hospitals 49(17), 46 (Sept. 1975).

4. J. Gettelshon and A. Wennberg, Small area variations in health care delivery. Science 182, 1102-1108 (Dec. 1973).

5. R. L. Bashshur, G. W. Shannon and C. A. Metzner, Some ecological differentials in the use of medical services. Hlth Services Research 6, 61 (1971).

6. A. Donabedian, Aspects of Medical Care Administration, Harvard University Press, Cambridge, Mass. pp. 419-425 (1976).

7. J. Studnicki, The minimization of travel efforts as a delineating influence for urban hospital service areas. Int. J. Hith Services 5, 679-693 (1975).

8. R. L. Morrill and R. Earickson, Locational efficiency of Chicago hospitals: An experimental model. Hith Services Research 4, 128-149 (Summer 1969).

9. D. L. Drossness and J. Lubin, Planning can be based on patient travel. Modern Hospital 106(4), $92-94$ (April 1966).

10. J. R. Griffith, Measuring Hospital Performance. Inquiry Book, Blue Cross Assoc., Chicago (1978).

11. E. J. Harner and P. B. Slater. Identifying medical regions using hierarchical clustering. Social Science and Medicine 14D, 3-10 (1980).

12. R. D. Coughlin, Hospital Complex analysis: an approach to analysis for planning a metropolitan system of service facilities. Doctoral Dissertation. University of Pennsylvania (1964).

13. H. D. Cherniack and J. B. Schneider, A new approach to delineation of hospital service areas. Discussion Paper Ser. No. 16, Regional Science Research Institute, Philadelphia (August 1967).

14. J. Schneider, Measuring locational efficiency of the urban hospital. Hith Services Research 2, 154 (Summer 1967).

15. P. deVise, Hospital study districts for metropolitan Chicago:
A geographic analysis and methodology, Geog. Anal. 2, 283 (July 1970).

16. E. Poland and P. Lemcke, Delinearion of Hospital Service Districts: Methodology and Statistical Appendices. Community Studies. Inc., Kanas City (1962).

17. A. Ciocco and I. Altman, Medical service area as indicated by intercounty movement of patients, Part I, Public Health Monograph No. 19, PHS No. 248 (Wash., D.C.: U.S. Government Printing Office, 1954).

18. Citizens Hospital Study Committee. Hospitals and Their Use in Northeastern Ohio. Citizens Hospital Study Committee, Cleveland (1961).

19. Transaction Systems, Inc., Evaluation of Alternative Health Area Definition Methods. Prepared for Bureau of Health Manpower, National Technology Information Service PB260672 (1976).

20. J. W. Thomas, Techniques for defining geographic boundaries for health regions, Socio-Econ. Plan. Sci. 13, 321-326 (1979).

21. F. G. Dickinson, Distribution of Physicians by Medical Service Areas, Bulletin 94, Bureau of Medical Economic Research, p. 9, AMA, Chicago (1954).

22. J. W. Mountin, E. H. Pennell and V. M. Hoge, Health Service Areas, Public Health Bulletin No. 292, Federal Security Agency, U.S. PHS. Wash: D.C., U.S. Government Printing Office (1945).

23. Government Studies and Systems, Inc. Methods to Determine Geographic Population Boundaries for Specific Health Services. NTIS-HRP-0900357/SWW (June 1978).

24. J. D. Taliaferro and W. W. Remmers, Identifying integrated regions for health care delivery. Hith Services Rep. 88, 337343 (1973).

25. D. H. Rosenbaum, A determination of health service areas of the eight hospitals in Volusia county, Florida. Washington University School of Medicine (1977).

26. L. R. Ford and D. R. Fulkerson, Flows in Networks, pp. 3, 17-22. Princeton University Press, Princeton, New Jersey (1962).

27. N. T. J. Baily, Statistics in hospital planning and design, Appl. Statist. 5, 146-157 (Nov. 1956). 